\title{
¿UVAS O RACIMOS? EJERCICIO DE METAFÍSICA
}

\author{
GRAPES OR BUNCHES? METAPHYSICS EXERCISE
}

CARLOS MuÑoz GuTIÉRREZ

Madrid

\section{RESUMEN}

En este trabajo se presenta, de modo didáctico, la manera en la que el autor concibe la metafísica como disciplina fundamental de la filosofía.

Palabras clave: metafísica, filosofía, sistema conceptual, forma de vida.

\section{ABSTRACT}

This paper presents, in a didactic way, the way in which the author conceives metaphysics as a fundamental discipline of philosophy.

Keywords: Metaphysics, Philosophy, conceptual system, way of life.

Llevo unos días preguntándome si lo que hay son uvas o racimos. Por un lado, lo básico, lo atómico, si iniciamos un proceso de descomposición hacia lo simple obtendríamos, sin lugar a dudas, uvas. Luego, podríamos afirmar que lo que realmente es, lo que constituye la condición del hecho de que exista algo, son las uvas, pues sin ellas no tendríamos en el fondo nada, o desde luego nada de interés. Ahora bien, si atendemos al proceso genético, al modo en el que lo simple, lo básico, lo que ya es indivisible, sin pérdida de su ser, llega al ser, sin duda encontramos el racimo que es su condición. Porque las uvas emergen en el crecimiento de los tallos que arracimados culminan en los frutos que son las 
uvas. Luego no puede existir la uva sin el racimo y, en consecuencia, lo que verdaderamente es, pues es la condición, es el racimo.

Pero, ¿qué es un racimo? Una relación de uvas, podríamos responder, un agrupamiento de uvas por las que éstas se relacionan y se vinculan. Porque tampoco el racimo sin uvas es nada, o nada de interés, un hombre desnudo, jugaba de pequeño con mi hermana una vez que nos habíamos comidos las uvas. Así que el racimo, aun siendo la condición necesaria para que existan uvas, no es nada sin ellas. Cuando esta doble condición ocurre, hablamos de una relación. Por consiguiente, lo que verdaderamente existe es la relación entre el tronco leñoso que conforma el racimo de donde penden las uvas. Luego, ni uvas ni racimo, sino la relación que las uvas establecen para conformar un racimo es lo verdaderamente real.

Una vez decidido que lo que existe es una determinada relación entre un conjunto de uvas que forman un racimo, podremos avanzar y atender con más detalle a qué tipo de relación se da cuando hablamos de racimo. ¿Es una relación por la que una uva se relaciona con todas las demás o con solo algunas de ellas? Parece que el racimo, como un fractal, crece creando más racimos. ¿Será que lo simple, lo atómico, no es tan atómico sino más bien molecular y que en realidad el ser de un racimo es el agrupamiento molecular de pequeños racimos? Es decir, la relación por la que las uvas conforman racimos es una relación molecular en donde un pequeño racimo de uvas se vincula a través de un tronco, de un eje común para formar un racimo mayor. No se relacionan todas las uvas entre sí, sino que un conjunto de uvas se relaciona con otros conjuntos de uvas y es por eso que hablamos de racimo. ¿Son las moléculas de uvas, es decir, pequeños racimos, entonces, lo verdaderamente existente?

Indudablemente ésta parece la mejor respuesta, atendiendo a su estructura, a su génesis, a la relación que se da entre las uvas, y a cualquier proceso de análisis que queramos realizar. Lo que existe son moléculas de uvas. La uva no puede darse de modo aislado, el racimo define una relación que consiste en que pequeños agrupamientos de uvas se unas unos con otros.

Pero, ¿no salen los racimos de las vides? En el fondo los racimos de uvas son el fruto de una planta que en su crecimiento produce las uvas. La vid es la condición necesaria para que exista la uva, luego lo verdaderamente real, la realidad del mundo objetivable es la vid. La vid es el nombre que damos a una planta que muestra determinadas características diferenciales frente a otras plantas, aunque también tiene otras propiedades comunes a toda una categoría, las plantas. Y aunque esto es así, hay otra singularidad, y es que la vid es el resultado de una intervención ajena a ella misma, porque si no, la vid se convierte en 
parra. Una parra es una vid que no ha sufrido podas artificiales. Luego la realidad natural verdaderamente existente es la parra. Aunque después de un rato de usar la expresión 'verdaderamente existente' me pregunto si una parra dibujada no es verdaderamente existe. Bueno, es una representación — podríamos aducir. Y las representaciones, aunque también son seres que entran a formar parte del mundo, su ser depende del ser representado.

El caso es que yo lo que quiero es vino... y volvemos a empezar.

La tradición que denominamos filosofía, podríamos decir, es una forma de saber que intenta conocer cómo es el mundo, en tanto que mundo, para determinar cómo podemos vivir en el felizmente. En el fondo, lo que me interesa del análisis de las uvas y del racimo es que de ellas obtenemos vino, que no solo en él encontramos la verdad, sino cierto estado alegre que nos eleva de modo diverso y según las circunstancias a un estar feliz en el mundo que, menos mal, produce vides que dan racimos de uva y que por un largo proceso...obtenemos vino.

¿Pero las vides o las parras no crecen en la tierra?

La filosofía vista de este modo se compone de dos grandes disciplinas o intereses, porque ya se habrá podido deducir que en todo lo anterior subyace una creencia o una forma de comprender el saber como el resultado de necesidades de los seres vivos. Los saberes buscan responder a problemas y los problemas demandan saberes. Todo ser vivo necesita un saber, saber lo que le conviene. Este es el axioma en el que se fundamenta esta - digamos - reflexión. Este ejercicio de reflexión, mejor.

Los seres vivos se encuentran con problemas en su objetivo fundamental de permanecer vivos, para solucionar estos problemas requieren de un saber que les diga cómo comportarse ante tal o cual dificultad que se les presenta en su camino. Este saber puede venir dado innatamente en repertorios genéticamente adquiridos a lo largo del proceso evolutivo de las especies, puede ser revelados por ser superiores que ya disponen de una solución al problema, o, puede que ni se tenga el saber ni nadie que lo enseñe y se tenga que afrontar la frecuentemente difícil tarea de producir un saber que nos proporcione una solución al problema. Esto es la vida.

La filosofía define una tradición de forma de saber para unos seres que se encuentran en el mundo ignorantes y arrogantes. Ignorantes porque no disponen del saber y arrogantes porque no quieren escuchar la solución revelada de ningún otro ser que no sean ellos mismos. Este saber quiere conocer el "verdadero" ser del mundo para deducir cómo actuar en él. A este saber, que es más un deseo, 
que no es más que una pretensión, que no puede verificarse sino entre los propios deseos de estos seres, es lo que hemos denominado filosofía. Y, visto así, la filosofía es el proyecto, inacabable, de relacionar el mundo que habitamos con nuestra tendencia a vivir bien en él. Metafísica y Ética son las dos grandes disciplinas tradicionales en las que la filosofía naturalmente se divide. ¿Qué es el mundo, cómo es el mundo, para que pueda ser feliz en él?

Ahora bien, ¿Qué significa conocer el mundo? Significará fundamentalmente responder para cada objeto, conjunto de objetos, situación o acción a las preguntas: ¿Qué es? ¿Qué clase de cosa es? ¿De qué está compuesto? ¿Cómo se comporta? ¿Cuáles son sus condiciones de existencia? ¿A qué otros elementos del mundo condiciona? ¿Con cuáles otros se relaciona? O, muy al contrario, conocer algo significa poder responder a una cuestión más interesada: ¿Para qué me puede servir? ¿Será útil o dañino, bueno o malo?

La segunda pregunta, que es la más importante de responder para nuestra supervivencia, requiere obtener una respuesta a la primera; porque para asegurarnos una vida tranquila en la que los peligros que el mundo esconde no nos asalten constantemente, necesitamos un saber que nos permita prever lo que va a pasar, pero también lo que pasará ante tales o cuales acciones que hagamos en el mundo, es decir, conocer significa poder prever las consecuencias de nuestros actos y la de los demás elementos de la realidad.

Como el mundo es muy grande, está lleno de elementos y además no se explica por sí mismo necesitamos determinadas estrategias de selección, agrupamiento y relación de los elementos del mundo que conforman nuestra experiencia, de categorización de lo que el mundo nos presenta. Adviertan que en la reflexión inicial sobre las uvas y los racimos, los átomos o las moléculas, la condición y lo condicionado nuestro problema no era realmente si lo que existen son uvas o racimos, sino más bien era determinar si es mejor ordenar el mundo en función de las cosas o de sus relaciones; de lo simple o de lo complejo; de la condición o de lo condicionado.

Antes que las uvas o los racimos, para poder ordenar el mundo, necesitamos de una estructura conceptual básica, que no forma parte del mundo, sino que es con ella con la que vamos a tener un mundo. Con esa estructura vamos a poder hacer una serie de cosas fundamentales para que el mundo pueda empezar a ser conocido, entre las cuales están:

1. Orientarnos espacio-temporalmente en el mundo. Tenemos que concebir el espacio y el tiempo como medidas del curso de los acontecimientos y para poder situarnos en la realidad. 
2. Generar una lógica causal con la que podamos establecer relaciones en ese curso temporal de los acontecimientos.

3. Determinar a qué vamos a considerar un objeto, una relación, un conjunto, una secuencia, una sucesión. Con esto podremos clasificar las cosas del mundo en categorías de objetos, especies o clases y, después, aprender sus características.

En general, antes de poder conocer el mundo, sus cosas, su funcionamiento y la utilidad que éstas tienen para nosotros tenemos que establecer criterios muy generales sobre qué es una cosa, qué es conocerla, qué es lo útil o lo bueno para nosotros, etc. Si no dispusiéramos de estos conceptos generales que orientan nuestro conocimiento del mundo y nuestra acción en él, nuestra vida sería como la de la mayoría de los animales.

Llamamos Filosofía, o más concretamente Metafísica, a la producción de estos conceptos generales con los cuales ponemos orden en el mundo según nuestro interés en él y por el que podemos conocer lo que pasa y lo que pasará ante tales o cuales acontecimientos.

Entre los conceptos fundamentales que produce la filosofía con los cuales respondemos a los problemas básicos e iniciales que el mundo nos presenta, según acabamos de ver, están los siguientes:

1. ¿Qué es el espacio y el tiempo?

2. ¿A qué vamos a considerar un objeto? Si observamos la realidad o lo que nuestra percepción visual nos ofrece de esta realidad, el mundo es un continuo de realidad. Los árboles están unidos a la tierra, los peces fluyen en el continuo del mar. ¿Dónde empieza una cosa y termina otra? Además entre el árbol y la tierra se establecen relaciones inseparables para que el árbol sea árbol, ¿debemos pensar el mundo como un conjunto de cosas o como un sistema de relaciones? Y si hay cosas, ¿de qué se componen? ¿Cuáles son los componentes elementales de la realidad? ¿Hay cosas materiales y espirituales?

3. Además qué características generales muestran las cosas del mundo: ¿puede una cosa dejar de ser lo que es? ¿Puede cambiar y seguir siendo lo mismo? ¿Pueden ser dos cosas a la vez? ¿Debe tener un lugar o posición o puede ocupar varios lugares a la vez? ¿Puede ser algo justo lo contrario de lo que es?

4. ¿Qué significa conocer las cosas del mundo y cómo podemos asegurar nuestro conocimiento de las mismas? ¿Para qué queremos 
conocerlas y hasta que nivel de profundidad? ¿Cómo vamos a expresar nuestro conocimiento y a organizarlo para que nos sea útil e interesante?

5. ¿Qué es lo bueno para nosotros? ¿Qué es lo que nos conviene hacer con el mundo y entre nosotros mismos? ¿Cómo vamos a organizar nuestra vida en el mundo? Y, sobre todo, ¿en qué medida nuestra comprensión de cómo es y de cómo funciona la realidad va a determinar la vida que podemos llevar en el mundo?

Como vemos antes de que podamos dar un paso en el mundo, nos asaltan innumerables dudas, muchos problemas que tenemos que resolver antes de hacer algo con el mundo, pero las soluciones no las encontramos en la realidad, sino que dependen de nosotros en la medida en que somos inteligentes y conscientes. Responder a estos problemas es la tarea de la filosofía y lo hace creando conceptos.

Por eso Gilles Deleuze y Félix Guattari definen la filosofía como el arte de crear conceptos. "Crear conceptos siempre nuevos, tal es el objeto de la filosofía (...). Los conceptos no nos están esperando hechos y acabados como cuerpos celestes. No hay firmamento para los conceptos. Hay que inventarlos, fabricarlos o más bien crearlos, y nada serían sin la firma de quienes lo crean."1

Pero si la filosofía es el saber que crea los conceptos básicos con los que ponemos orden en el mundo permitiéndonos orientar nuestra acción en él, inmediatamente surgen innumerables cuestiones.

Por ejemplo, ¿Los conceptos que creamos pueden juzgarse como verdaderos o falsos? O dicho de otra manera ¿es la filosofía una ciencia y podemos evaluar sus conceptos como hacemos con las teorías científicas? ¿Hay una filosofía verdadera y definitiva?

La filosofía no es una ciencia porque no estudia nada en concreto, sino que dispone los recursos conceptuales con los cuales podemos hacer ciencia. La ciencia estudia el mundo, pero una vez que hemos decidido que hay un mundo y que hay cosas en él que podemos estudiar. Naturalmente, a la hora de hacer ciencia, la filosofía siempre está presente en la base de los conceptos básicos de la ciencia, en su creación, en su definición. Pero también está presente en la base de cualquier faceta de la actividad humana, porque siempre hay conceptos que nombran, definen y dirigen lo que hacemos en el mundo, incluso en la comprensión de nosotros mismos. Pero no hay forma de evaluar los conceptos que la filosofía crea, salvo respecto a la utilidad o la conveniencia que puedan tener

1 Gilles Deleuze y Félix Guattari. Qu'est-ce que la philosophie?, (Les Éditions de Minuit, Paris, 1991) traducción de Thomas Kauf, ¿Qué es la filosofia?, (Anagrama, Barcelona, 1993) 11. 
para las vidas humanas. Los conceptos que crea la filosofía se pueden sustituir en su contenido dependiendo de la manera de analizar y de comprender los hechos que el mundo nos presenta. Ver cómo una determinada comprensión nos permite determinada forma de vida o determinadas prácticas, pero no hay nada previo a estos conceptos básicos con los que pudiéramos evaluarlos o juzgarlos. Por eso también Deleuze define la filosofía de la siguiente manera:

"La filosofía no comunica nada especial. Solo permite realizar encuentros y ver ciertas cosas. Sirve como los lentes de Spinoza. Si ves algo, adelante, $\mathrm{Si}$ no, tíralos y coge otros. En la filosofía no hay nada que comprender. Ella no preexiste ni conduce a ninguna prescripción. La filosofía es una relación física con los demás y la invención de un modo de vida."

Hasta aquí, creo, todo está claro, resulta simple y elegante. Sobrevivir en el mundo es un problema para los distintos seres que lo pueblan. Hay que producir un saber que nos permita saber lo que va a pasar y, sobre todo, anticipar las consecuencias de lo que pasó o de lo que está pasando. Este saber requiere de uno previo, fundamental, por medio del cual ordenamos el mundo, ponemos las cosas en un sitio. Este saber no forma parte del mundo, o lo tenemos o se nos revela o lo elaboramos. Pero, cuando lo elaboramos y somos conscientes que es un saber producido por nosotros mismos la cosa se complica, y mucho.

Tenemos problemas, necesitamos un saber para solucionarlos, y el saber que nos permite solucionar nuestros problemas contiene precisamente aquello que identifica el problema y el plan general para su solución. Identificar los problemas es el punto de partida para ponerles solución, a partir de aquí va a nacer una espiral problemática. ¿Quiénes tienen problemas? ¿Para quiénes sirven las soluciones? Y la solución a un problema para algunos, ¿no crea un problema a otros? Tengo hambre, puedo solucionar este inconveniente cazando un animal, y así, el animal se encuentra con un problema.

¿Hay algún modo de ordenar el mundo que nos permita solucionar todos los problemas que el mundo nos presenta a todos los seres que lo pueblan? En la universalidad, en el 'todos' contenido en nuestra última cuestión está el foco de lo que hemos llamado filosofía. Pero incluso esta pretensión nació mucho después de que ya existieran en el mundo multiplicidad de saberes que solucionaban algunos problemas a algunos seres, saberes adquiridos en el proceso evolutivo de determinadas especies, saberes revelados por dioses variados que habían establecido alianzas singulares con determinados grupos humanos, saberes producidos para satisfacer intereses muy concretos. Y los saberes crean mundo y proporciona poder a quienes lo tienen y en un mundo ordenado por multiplicidad de saberes diversos aparece una pretensión humana que incorpora una 
universalidad, seguramente insensata, pero también evidente. Por eso resulta que:

"Los filósofos ya no deben darse por satisfechos con aceptar los conceptos que se les dan para limitarse a limpiarlos y a darles lustre, sino que tienen que empezar por fabricarlos, crearlos, plantearlos y convencer a los hombres de que recurran a ellos. Hasta ahora, en resumidas cuentas, cada cual confiaba en sus conceptos como en una dote milagrosa procedente de algún mundo igual de milagroso."

Efectivamente, para que el mundo cambie, para que nuestra relación con la realidad, nuestra organización social o política, los tipos de relaciones que tenemos entre nosotros y con el resto de los seres vivos cambie, alguien debe mirar de otra manera lo dado, lo existente. Alguien debe cuestionar los conceptos con los que hemos edificado nuestro estar en el mundo, porque, ¿a quién le gusta por completo lo que se encuentra en la realidad? Y aunque solo sea porque el hombre es un ser activo, que constantemente desea cosas y quiere hacerlas realidad, debemos siempre pensar cómo llevar a efecto nuestros deseos. Cómo hacer que aquello que nos gustaría que fuera, ocurra en el mundo. Para todo eso, siempre tenemos que pensar con qué criterios y con qué normas debemos actuar, qué valores van a dirigir nuestras acciones.

Puesto que siempre estamos abiertos al mundo y éste recibe nuestra acción en él, debemos siempre replantear los conceptos con los que comprendemos lo existente, los criterios con los que vamos a actuar y los valores que rigen nuestro comportamiento. Eso fundamentalmente es la tarea de la filosofía. Crear y recrear constantemente los conceptos con los que ordenamos el mundo. Cada época, cada situación de la historia, las condiciones cambiantes de la realidad, las expectativas de los hombres y las capacidades que su conocimiento les aporta, exige que sean revisados los conceptos y valores con los que hemos construido el mundo humano. Por eso, la filosofía siempre es historia de la filosofía.

La filosofía, que, como hemos dicho, es básicamente construir un modo de vida, no puede evaluarse y en consecuencia siempre está en progreso, es más, casi estamos obligados a no parar de pensar, porque solo así las cosas pueden mejorar. Por eso, la filosofía es un saber crítico, porque no solo crea los conceptos que nos permiten poner orden en el mundo, sino que constantemente los está revisando para ir ajustándolos a los resultados y a los deseos universales que los seres humanos quieren extender por el mundo. Hay filosofía en la medida en que el ser humano desea y como desear es intentar que lo deseado se haga

2 Friedrich Nietzsche. Fragmentos Póstumos 1884-1885. sobre «el arte de la desconfianza». Citado de Deleuze y Guattari, ibid. 11. 
realidad, la filosofía siempre está a la base de lo que somos y de lo que podemos llegar a ser, por eso, quizá, la definición más completa de lo que sea la filosofía es la que podemos encontrar en las siguientes palabras de Michel Foucault:

"La Filosofía es el movimiento por el que, no sin esfuerzo y tanteos y sueños e ilusiones, nos desprendemos de lo que está adoptado como verdadero y buscamos otras reglas de juego. La filosofía no es sino el desplazamiento y la transformación de los marcos de pensamiento; la modificación de los valores recibidos y todo el trabajo que se hace para pensar de otra manera, para hacer algo otro, para llegar a ser otra cosa que lo que se es..."3

El espacio propio de la filosofía es el que media entre lo que todavía no es, pero desearíamos que fuera y lo que es y nos gustaría cambiar. Al pensar cómo podemos producir tales o cuales cosas, o al planear nuestras acciones, también al evaluar si nos gusta lo que hay, necesitamos a la filosofía. Necesitamos criterios de valoración y de acción, necesitamos valores y normas que no nos equivoquen, o que lo hagan lo menos posible, al intervenir en la realidad, al crear nueva realidad o al decidir cuál será el mejor curso de acción para nosotros.

Esta es la tarea primordial de la Filosofía: evaluar lo que hay y determinar los criterios mediante los cuales podremos mejorar la realidad.

Visto así, la filosofía resultaría una suerte de ingeniería, para evitar esta deformación, debemos unir a esta explicación su dimensión humanista. Es decir, lo que aporta la filosofía no son diseños de artefactos o de máquinas, sino valores de por qué realizarlas y de cómo emplearlas.

La investigación filosófica se centra en la creación, el análisis y la revisión racional de los valores fundamentales con proyección universal que orientan la vida humana: el ser, la verdad, el bien, la virtud, la justicia, la belleza, etc. Y a partir de esta reflexión elabora un discurso metódico, razonado y crítico que evalúa lo que hay y sugiere cómo transformarlo.

Queremos vino que nos alegre, pero que no emborrache, que su producción no suponga el fin de la tierra que es su fuente, ni la explotación de las manos que lo elaboran, que podamos disfrutarlo todos y para siempre. Si esto queremos, si es una pretensión compartida, ¿qué es lo real? ¿Las uvas o los racimos?

3 M. Foucault. "Le philosophe masqué" (entrevista con C. Delacampagne, Febrero de 1980), Le Monde, $\mathrm{n}^{\circ}$ 10945, 6 Abril de 1980, Le Monde-Dimanche, pp. I y XVII. 


\section{REFERENCES}

Deleuze, Gilles, Guattari, Félix. Qu'est-ce que la philosophie?. Paris: Les Éditions de Minuit, 1991 (ed. española ¿Qué es la filosofia?. Traducido por Thomas Kauf. Barcelona: Anagrama, 1993).

Carlos Muñoz Gutiérrez

Madrid (España)

https://orcid.org/0000-0001-6734-4615 